\title{
In-situ Observation of Structure and Conductance Evolution of Gold Contact during Stretching
}

\author{
Y. Oshima***, Y. Kurui*** and K. Takayanagi*** \\ * Dep. Mat. \& Sci. Eng., Tokyo Tech., J1-3, 4259 Nagatsuta, Midori-ku, Yokohama, 226-8502, \\ Japan. \\ ** JST, PRESTO, 5-Sanbancho, Chiyoda-ku, Tokyo, 102-0075, Japan \\ *** Dep. Phys., Tokyo Tech., 2-12-1-H-51 Oh-okayama, Meguro-ku, Tokyo 152-8551, Japan
}

Metallic contacts have attracted much interest because of quantized conductance at room temperature. It has been investigated mainly by conductance histogram of many stretching processes. For example, gold contact shows $1,2,3,4$ times the quantized unit of conductance $\left(\mathrm{G}_{0}=2 e^{2} / h\right.$ where $e$ is the elementary charge and $h$ is Planck's constant) in the histogram. However, each stretching process does not always show the same conductance values. It seemed to relate to variation of structural evolution, but there was no evidence. In this study, we investigate structure and conductance evolution of gold contact which axis is the [110], [100] or [111] direction during stretching using a ultra high vacuum transmission electron microscope (UHV-TEM) combined with a scanning tunneling microscope (STM). We found that the evolution of gold [110] contact is different from gold [100] or gold [111] contact.

A gold contact was formed in the TEM, and then stretched by the piezo drive of the STM, as described elsewhere [1]. The mechanical stability of the contact, TEM images were recorded every $33 \mathrm{~ms}$, simultaneous with the conductance measurements. The conductance was measured every $0.033 \mathrm{~ms}$ (30 kHz sampling rate), with a bias voltage of $13 \mathrm{mV}$. The conductance values did not depend upon whether the incident electron beam irradiated the specimen or not [1]. Fabrication of the contacts was performed by repeating an attaching-breaking cycle for the two electrodes. The evolutions of structure and conductance were compared among the [110], [100] and [111] orientations. Variations in the stretching speed during stretching, in the range of $0.1-7.3 \mathrm{~nm} / \mathrm{s}$, had no noticeable effect. The stretching speeds were similar to those used in STM experiments, but much slower than those used in simulations (on the order of $1010 \mathrm{~nm} / \mathrm{s}$ ). The displacement of the electrode by the piezo drive was calibrated by TEM images to be $4.4 \mathrm{pm}$ per digital bit.

Figure 1 shows conductance evolution of gold [110], [100] and [111] contacts during stretching. Gold [110] contact of Fig. 1(a) had a uniform hexagonal prism cross-section [2]. Trace A had rather flat plateaus at $\mathrm{G}_{0}$ multiples of $9,8,7,3,2$, and 1 , while trace $\mathrm{B}$ had plateaus at $\mathrm{G}_{0}$ multiples of 9,8 , 7 , and 3,2 , and 1 . The conductance traces had no conspicuous decrease in the elastic regime, but leveled off near integer multiples of the quantized unit of conductance. The length of the plateaus was in the range of 0.05-0.025 nm. Gold [100] contact of Fig. 1(b) had a short bottleneck and suspended between two quadrangular pyramid-shaped electrodes surrounded by four $\{111\}$ facets. Both traces A and B were similar, and had gradual slopes beginning around 10,7 , and $4 \mathrm{G}_{0}$. In detail, the conductance slopes beginning at 10 and $7 \mathrm{G}_{0}$ decreased gradually, and then jumped at each 0.2 nm elongation, which was the spacing of the (100) lattice plane. Gold [111] contact of Fig. 1(c) had a short bottleneck and suspended between two truncated triangular pyramid electrodes surrounded by three $\{111\}$ and three $\{100\}$ facets. The trace showed gradual slopes beginning at 10,7 , and $5 \mathrm{G}_{0}$. and the conductance jumped at each $0.24 \mathrm{~nm}$ elongation, which was the spacing of the (111) lattice 
plane. We found that gold [111] and [100] contacts show very similar behavior during stretching, and they were different from gold [110] contact.

Figure 2 shows structure and conductance evolution of gold [111] contact. As shown in Fig. 2(a), gold [111] contact was elastically stretched until a single (111) lattice plane was introduced by plastic deformation. Between the plastic deformations, it was stretched by approximately $0.24 \mathrm{~nm}$, which corresponds to the spacing of the (111) lattice. We concluded that the contact axis is a key parameter in the stretching process.

[1] Y. Oshima, K. Mouri, H. Hirayama and K. Takayanagi, Surf. Sci. 531 (2003) 209.

[2] Y. Kurui, Y. Oshima, M. Okamoto and K. Takayanagi,Phys. Rev. B. 79 (2009) 165414.

(a)

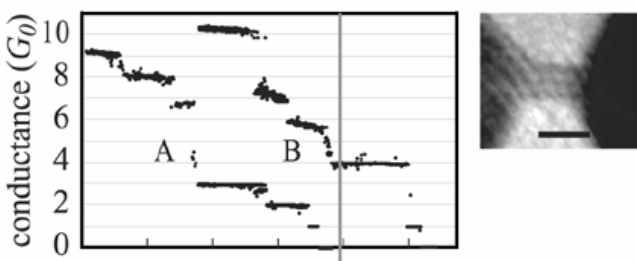

(b)

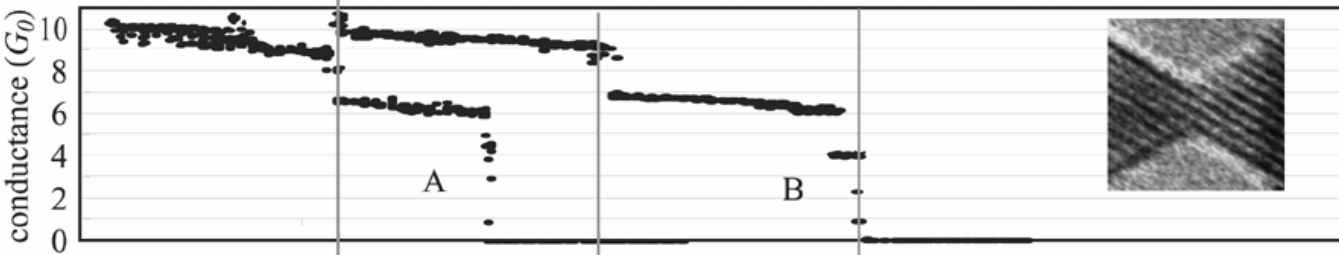

(c)

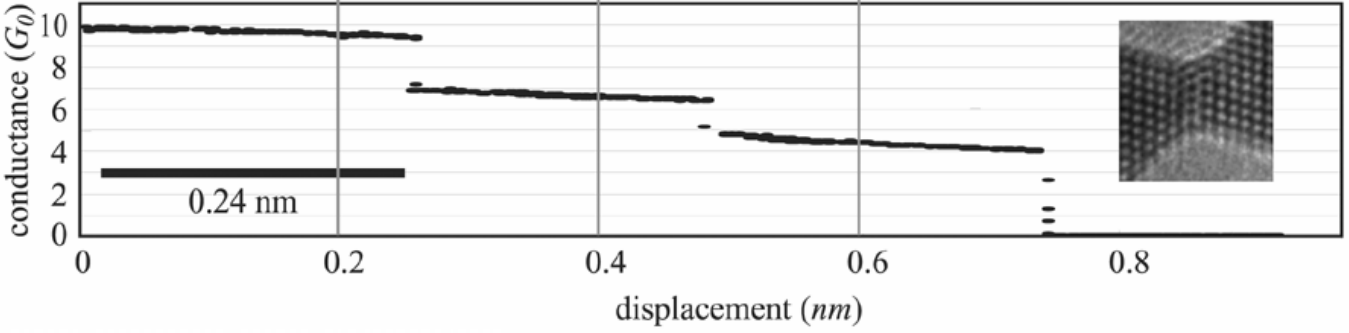

(a)
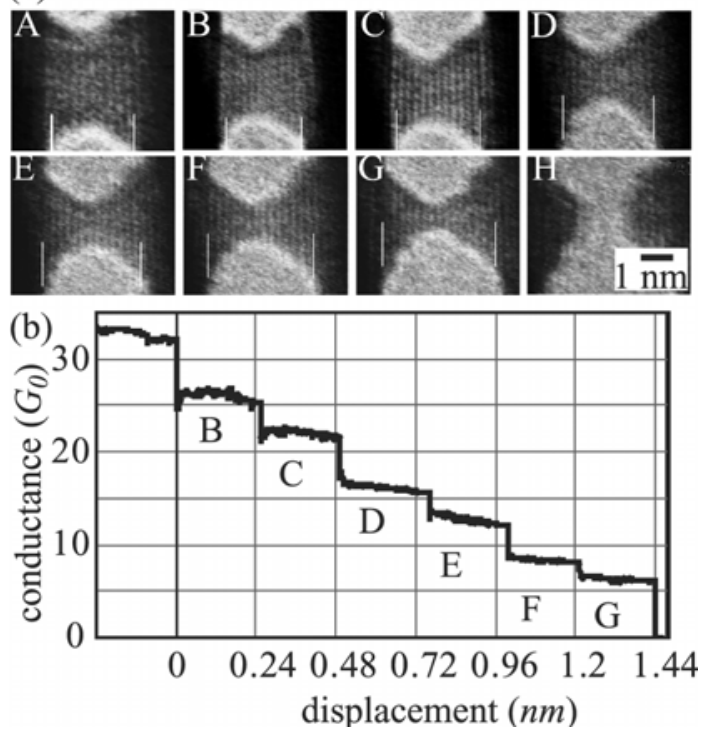

Fig. 1 Conductance evolutions and typical TEM images of gold (a) [110], (b) [100] and (c) [111] contacts during stretching, respectively.

Fig. 2 (a) A series of TEM images taken after the abrupt conductance jumps. (b) Conductance evolution during stretching of a gold [111] contact. The gradual decrease and abrupt jumps correspond to elastic and plastic deformations, respectively. 